\title{
Fatty acid translocase promoted hepatitis B virus replication by upregulating the levels of hepatic cytosolic calcium
}

Jian Huang ${ }^{1}$, Lei Zhao ${ }^{1}$, Ping Yang ${ }^{1}$, Zhen Chen ${ }^{1}$, Xiong Z. Ruan ${ }^{1,2,3}$, Ailong Huang ${ }^{1,3}$, Ni Tang $^{1, *}$, Yaxi Chen ${ }^{1, *}$

${ }^{1}$ Centre for Lipid Research \& Key Laboratory of Molecular Biology for Infectious Diseases (Ministry of Education), Institute for Viral Hepatitis, Department of Infectious Diseases, the Second Affiliated Hospital, Chongqing Medical University, Chongqing 400016, China; ${ }^{2}$ John Moorhead Research Laboratory, Centre for Nephrology, University College London Medical School, Royal Free Campus, University College London NW3 2PF, United Kingdom. ${ }^{3}$ The Collaborative Innovation Center for Diagnosis and Treatment of Infectious Diseases (CCID), Zhejiang University, Hangzhou 310058, China.

Abbreviations: SOC, store-operated $\mathrm{Ca}^{2+}$; WT, wild type; CD36KO, CD36 knockout; CD36OE, CD36 overexpression; shCD36, shRNA-targeting CD36; shCont, shRNA-scramble control.

*Corresponding author. E-mail address: chenyaxi@cqmu.edu.cn (Y.Chen) and nitang@ cqmu.edu.cn (N. Tang). 


\begin{abstract}
Hepatitis B virus (HBV) is designated a "metabolovirus" due to the intimate connection between the virus and host metabolism. The nutrition state of the host plays a relevant role in the severity of HBV infection. Metabolic syndrome (MS) is prone to increasing HBV DNA loads and accelerating the progression of liver disease in patients with chronic hepatitis B (CHB). Cluster of differentiation 36 (CD36), also named fatty acid translocase, is known to facilitate long-chain fatty acid uptake and contribute to the development of MS. We recently found that CD36 overexpression enhanced HBV replication. In this study, we further explored the mechanism by which CD36 overexpression promotes HBV replication. Our data showed that CD36 overexpression increased HBV replication, and CD36 knockdown inhibited HBV replication. RNA sequencing found some of the differentially expressed genes were involved in calcium ion homeostasis. CD36 overexpression elevated the cytosolic calcium level, and CD36 knockdown decreased the cytosolic calcium level. Calcium chelator BAPTA-AM could override the HBV replication increased by CD36 overexpression, and the calcium activator thapsigargin could improve the HBV replication reduced by CD36 knockdown. We further found that CD36 overexpression activated Src kinase, which plays an important role in the regulation of the store-operated $\mathrm{Ca}^{2+}$ channel. An inhibitor of Src kinase (SU6656) significantly reduced the CD36-induced HBV replication. We identified a novel link between CD36 and HBV replication, which is associated with cytosolic calcium and the Src kinase pathway. CD36 may represent a potential therapeutic target for the treatment of CHB patients with MS.
\end{abstract}

Keywords: CD36; Hepatitis B virus; Replication; Cytosolic calcium; Src kinase 


\section{Introduction}

Hepatitis B virus (HBV) is a small DNA virus that predominately infects liver cells and is the most common cause of hepatitis, cirrhosis and hepatocellular carcinoma [1]. It is also designated a "metabolovirus" due to the intimate connection between the virus and host metabolism [2]. HBV transcription and replication is regulated mainly by the recruitment of a variety of cellular transcription factors to its promoters and enhancers, some of which are linked to host metabolic processes [3]. Several epidemiological studies demonstrated that the individual diet and host nutrition state play relevant roles in the severity of HBV infection [4]. Starvation and fasting increased HBV transcription and gene expression [5]. Bile acids also increased HBV transcription and gene expression in vitro experiments [6]. Host cholesterol was an essential element for HBV assembly and viral infection $[7,8]$. These results suggested that the host metabolic changes generate a permissive milieu that may be advantageous to the HBV life cycle.

The number of chronic hepatitis B (CHB) patients with concomitant metabolic diseases has increased gradually. A recent study reported that $13 \%$ of $\mathrm{CHB}$ patients had metabolic syndrome (MS), and the prevalence of liver cirrhosis was higher in CHB patients with MS (38\%) than those without MS (11\%) [9]. A population-based study showed that CHB patients with MS had significantly higher levels of HBV DNA loads compared to those without MS [10]. High viral load is considered an important risk factor for the development of liver cirrhosis and hepatocellular carcinoma in CHB patients. Therefore, further exploration of the hepatic metabolic factors that control HBV replication may be an important step in the treatment 
strategies for CHB patients with MS.

Cluster of differentiation 36 (CD36), also named fatty acid translocase, belongs to the scavenger receptor family and is a transmembrane glycoprotein found on the surface of many cell types in vertebrates [11]. It is a facilitator of long-chain fatty acid transport and other biologically active lipids, such as ox-LDL. With many ligands and functions, CD36 could have an influence on various conditions associated with MS, atherosclerosis and non-alcoholic fatty liver disease (NAFLD) [12]. The expression of CD36 was weak in the normal liver, but a mouse model showed that its expression was significantly increased during high fatty acid feeding [13] Clinical studies also exhibited higher soluble CD36 in patients with NAFLD and MS $[14,15]$. Recent studies demonstrated that a chronic HBV immune tolerant phase characterized by high levels of HBV replication had higher levels of free fatty acids compared with healthy controls [16]. In addition, elevated levels of total fatty acids are clearly induced in the HBV-replicating cell line HepG2.2.15 [17]. However, whether CD36 is a molecular bridge between fatty acid metabolism and HBV replication is largely unknown.

Our previous study found that the expression of CD36 was increased in the HBVreplicating cell line, and CD36 overexpression enhanced HBV replication, but the mechanism is still unknown [18]. In this study, we aimed to investigate the molecular mechanism by which CD36 overexpression enhanced HBV replication.

\section{Materials and methods}

\subsection{Cell culture and construction}


HepG2.2.15 and HepAD38 cells were cultured in modified Eagle's medium (MEM) containing $10 \%$ fetal calf serum and $500 \mu \mathrm{g} / \mathrm{mL}$ G418. To stably overexpress CD36 in HepG2.2.15 and HepAD38 cells, a human CD36 overexpression lentivirus system was constructed, packaged, and purified by GeneChem (Shanghai, China). CD36-overexpressionlentivirus and vector-lentivirus were transfected into HepG2.2.15 and HepAD38 cells according to the manufacturer's protocol. To generate CD36 knockdown cells, a lentivirus system expressing CD36 shRNA was constructed by GeneChem. The CD36 shRNA targeting sequence was as follows: 5'-GGCTGTGTTTGGAGGTATTCT-3'. CD36-shRNA-lentivirus and scramble control-lentivirus were transfected into HepG2.2.15 and HepAD38 cells according to the manufacturer's protocol.

2.2 Purification and quantification of core particles associated with $H B V D N A$ from $H B V$ replicating cells

Core particles associated with HBV DNA were extracted from equal numbers of cells 96 $\mathrm{h}$ after transfection. Cells were collected, and cell number was counted using an Innovatis Cedex XS Analyzer (Roche, Bielefeld, Germany). Next, cells were washed with PBS and lysed with $500 \mu \mathrm{L}$ of lysis buffer (50 mM Tris- $\mathrm{HCl}$ (pH 7.4), $1 \mathrm{mM}$ EDTA, $1 \%$ NP-40) at room temperature for $30 \mathrm{~min}$. Cell nuclei were pelleted by centrifugation for 1 minute at $10000 \mathrm{~g}$. Then, the supernatant was treated with $60 \mathrm{U}$ of DNase I and $10 \mathrm{mM} \mathrm{MgCl}_{2}$ for $4 \mathrm{~h}$ at $37^{\circ} \mathrm{C}$ and centrifuged again. The supernatant was supplemented with $200 \mu \mathrm{L}$ of 35\% PEG8000 containing 1.5 $\mathrm{M} \mathrm{NaCl}$ and rested on ice for 40 minutes. Then, the pellet of the virus core particles was collected after centrifugation at $12000 \mathrm{~g}$ for $5 \mathrm{~min}$ at $4^{\circ} \mathrm{C}$. The virus core particles were digested 
overnight at $42^{\circ} \mathrm{C}$ in $500 \mu \mathrm{L}$ digestion buffer $(0.5 \mathrm{mg} / \mathrm{mL}$ proteinase $\mathrm{K}, 25 \mathrm{mM}$ Tris- $\mathrm{HCl}(\mathrm{pH}$ 8.0), $0.5 \% \mathrm{SDS}, 150 \mathrm{mM} \mathrm{NaCl}$, and $10 \mathrm{mM}$ EDTA). HBV DNA in the digestion mixture was extracted twice with phenol-chloroform (1:1), precipitated with ethanol and glycogen, and dissolved in $\mathrm{ddH}_{2} \mathrm{O}$. HBV DNA was analyzed by southern blot (DIG-High Prime DNA Labeling and Detection Starter Kit II, Roche, Rotkreuz, Switzerland) and real-time PCR using the SYBR Green method (Takara, Dalian, China). The forward and reverse primers were as follows: $\quad 5^{\prime}-\quad$ TGCGGCGTTTTATCATATTCC $\quad-3^{\prime}$ and $5^{\prime}-$ ATACCTTGGTAGTCCAGAAGAACCA-3', respectively.

To determine the effect of chemical reagents on the process of CD36 affecting HBV replication, CD36 overexpression cells were treated with calcium chelator BAPTA-AM (Selleck, Houston, TX, USA) at a concentration of $25 \mu \mathrm{M}$ and Src family kinase inhibitor SU6656 (Selleck, Houston, TX, USA) at a concentration of $0.6 \mu \mathrm{M}$, and CD36 knockdown cells were treated with calcium mobilizing agent thapsigargin (Sigma-Aldrich, St. Louis, MO, USA) at a concentration of $5 \mathrm{nM}$ and $2.5 \mathrm{nM}$ in HepAD38 and HepG2.2.15, respectively. After $96 \mathrm{~h}$, the HBV DNA was extracted and measured by real-time PCR, as described above. Cell viability under each condition was assayed by using trypan blue.

\subsection{Measurement of the secreted HBsAg and HBeAg}

The concentrations of HBV surface antigen (HBsAg) and HBV e antigen (HBeAg) in the culture medium were detected by the enzyme-linked immunosorbent assay (ELISA) kit (KHB, Shanghai, China) according to the manufacturer's protocols. 


\subsection{Animal study}

To build the mouse model of acute hepatitis B virus infection, 9-week-old male C57BL/6 wild type (WT) and CD36 knockout (CD36KO) mice were used. CD36KO mice created on a C57BL/6 background were kindly provided by Dr. Maria Febbraio (Lerner Research Institute, U.S.). All animal studies were conducted according to the protocol approved by the Institutional Animal Care and Use Committee at Chongqing Medical University. A total of $20 \mu \mathrm{g}$ of pGEMHBV1.3 plasmid was injected into the tail veins of mice in a volume of saline equivalent to $8 \%$ of the mouse body weight within 5-8 s. The serum and liver tissue samples were collected on the fifth day. The levels of HBsAg and HBeAg were measured by chemiluminescence immunoassay (Abbott Architect, USA). HBV DNA in the serum and liver was extracted using the Hipure viral DNA mini kit (Magen, Guangzou, China) and measured by real-time PCR, as described above. The HBV core protein of liver tissue was visualized by immunohistochemical staining using anti-HBc antigen antibody (1:100, Abcam, Cambridge, UK).

\subsection{Cytosolic calcium analysis}

Cells were cultured in a 6-well plate. At $48 \mathrm{~h}$ posttransfection, cells were washed with DHanks buffer (without $\mathrm{Ca}^{2+}$ and $\mathrm{Mg}^{2+}$ ) two times and then incubated with $4 \mu \mathrm{M}$ fluorescent $\mathrm{Ca}^{2+}$ indicator Fluo-4/AM (life technologies, Grand Island, NY, USA) and $2.5 \mathrm{mM}$ probenecid (Life Technologies, Grand Island, NY, USA) at $37^{\circ} \mathrm{C}$ and $5 \% \mathrm{CO}_{2}$ in the dark for 30 minutes and then at room temperature for an additional 30 minutes. Cells were digested with trypsin and precipitated by centrifugation. Cell pellets were washed in cold D-Hanks buffer twice and resuspended in $500 \mu \mathrm{L}$ D-Hanks buffer. The concentration of intracellular calcium was 
measured using FACSCanto ${ }^{\mathrm{TM}}$ II flow cytometry (BD, FranklinLakes, NJ, USA) by excitation with a $488 \mathrm{~nm}$ argon laser line and was shown by mean fluorescence. Data were collected from more than 10,000 cells for each sample. The $\mathrm{Ca}^{2+}$ concentrations were expressed as relative fluorescence.

To determine the role of calcium reagents in reducing and elevating cytosolic calcium, CD36 overexpression cells were treated with calcium chelator BAPTA-AM at a concentration of $25 \mu \mathrm{M}$, and CD36 knockdown cells were treated with calcium mobilizing agent thapsigargin at concentrations of $5 \mathrm{nM}$ and $2.5 \mathrm{nM}$ in HepAD38 and HepG2.2.15, respectively. After $48 \mathrm{~h}$, the concentration of intracellular calcium was measured, as described above. Cell viability under each condition was assayed using trypan blue.

\subsection{Western blotting}

Cells were lysed using RIPA containing a protease inhibitor cocktail and phosphatase inhibitor cocktail, and the protein content was measured and normalized using a BCA protein assay kit. Total proteins $(50 \mu \mathrm{g})$ were separated by SDS-PAGE and transferred onto PVDF membranes. After blocking with $3 \%$ BSA, the membranes were incubated with primary antibodies: anti-CD36, 1:2000 (Abcam, Cambridge, UK); anti-Src, anti-p-Src, 1:2000 (CST, Danvers, MA, USA); anti-actin, 1:4000, (ProteinTech, Wuhan, China), at $4^{\circ} \mathrm{C}$ overnight and subsequently incubated with the corresponding horseradish peroxidase (HRP)-conjugated secondary antibodies. Finally, the blot was detected using an ECL advance western blotting detection kit (Millipore, Temecula, CA, USA). The protein signal intensity was analyzed using ImageJ software (National Institutes of Health, USA). 
Cells were cultured in a 10-cm plate. IP3 was extracted from equal numbers of cells at 72 h. IP3 levels in the cytoplasm were measured with a human IP3 ELISA kit (SinoBestBio, Shanghai, China).

\subsection{Statistics}

Experimental data are shown as the means \pm standard deviations. The t-test was applied to comparisons between different groups; values of $\mathrm{P}<0.05$ were considered to indicate statistical significance. Statistical analysis was performed using GraghPad Prism software.

\section{Results}

\subsection{CD36 overexpression promoted $H B V$ replication in vitro}

HepG2.2.15 and HepAD38 cells, which support stable HBV replication, protein expression, and virion assembly and secretion, were infected with recombinant lentivirus expressing CD36 cDNA to obtain stable CD36 overexpression (CD36OE) cell line models. There were significant increases in CD36 protein levels in the CD36OE group compared with in the vector group (Figure 1A), suggesting that CD36 overexpression was successfully established in both cell lines. HBV DNA replicative intermediates were detected by both realtime PCR and southern blot. The data showed that CD36 overexpression induced significantly elevated levels of HBV DNA in both cell lines (Figure 1B and 1C). Meanwhile, CD36 overexpression significantly elevated the levels of $\mathrm{HBsAg}$ and $\mathrm{HBeAg}$ compared with those in the vector group (Figure 1C). These data suggested that CD36 overexpression could enhance 
HBV replication.

3.2 CD36 knockdown inhibited HBV replication in vitro and in vivo

As shown in Figure 2A, CD36 expression was efficiently downregulated in shRNAtargeting CD36 (shCD36) compared with shRNA-scramble control (shCont) in both HepG2.2.15 and HepAD38 cells, suggesting that stable CD36 knockdown cell lines were successfully established. CD36 knockdown resulted in decreased levels of HBV DNA replicative intermediates in both cells (Figure 2B and 2C). Gene silencing of CD36 significantly inhibited HBsAg and $\mathrm{HBeAg}$ secretions (Figure 2C). These data suggested that CD36 knockdown inhibited HBV replication.

In an acute $\mathrm{HBV}$ infection mouse model, the serum HBsAg and HBeAg levels were significantly decreased in CD36 knockout (CD36 KO) mice compared with in wild type (WT) mice (Figure 2D). Moreover, the levels of HBV DNA in the serum and liver were remarkably decreased in CD36 KO mice compared with in WT mice (Figure 2D). The expression of HBcAg in livers from the two groups of mice was detected by immunohistochemical staining. The frequency of $\mathrm{HBcAg}$-positive hepatocytes decreased significantly in $\mathrm{CD} 36 \mathrm{KO}$ mice compared with in WT mice (Figure 2D). These data in vivo were similar to those in vitro.

\subsection{CD36 expression affected the levels of cytosolic calcium in vitro}

To explore the molecular mechanism by which CD36 affects HBV replication, we performed RNA sequencing of the mRNA from HepG2.2.15-CD36OE cells and HepG2.2.15vector cells to understand the differential transcriptomic profile. Our results showed that some 
of the differentially expressed genes, such as KCNK10, SCN9A, and S100A3, were involved in the potassium voltage-gated channel, sodium voltage-gated channel and calcium binding protein (Figure 3A). Next, we specially focus on the potential role of CD36 in calcium ion homeostasis, which supports HBV replication and assembly, as shown in previous studies $[19,20]$. We found that CD36 overexpression significantly increased the levels of cytosolic calcium and CD36 knockdown decreased the levels of cytosolic calcium, as evidenced by flow cytometry (Figure 3B and 3C), suggesting that CD36 expression affected the levels of cytosolic calcium in both HepG2.2.15 and HepAD38 cells.

3.4 Cytosolic calcium signals involved in CD36-mediated regulation of HBV replication in vitro

Furthermore, the data showed that the calcium chelator BAPTA-AM reduces cytosolic calcium in CD36 overexpression cells (Figure 4A), while the calcium mobilizing agent thapsigargin increases cytosolic calcium in shCD36 cells (Figure 4B). The levels of HBV DNA were decreased in the BAPTA-AM-treated CD36OE group compared with in the untreated group, and the levels of HBV DNA were increased in thapsigargin treated shCD36 cells (Figure 4A and 4B), suggesting that CD36 was implicated in HBV replication by cytosolic calcium.

\subsection{CD36 overexpression enhanced HBV replication by activating Src kinase}

CD36 has been linked to the phosphorylation of Src kinases, which play an important role in the regulation of calcium influx $[21,22]$. The activity of Src kinases is regulated by the phosphorylation at the Tyr416 site in the activation loop domain to upregulate enzyme activity [23]. We investigated the effect of CD36 overexpression on the activity of Src kinase in HepG2.2.15 and HepAD38 cells. The data demonstrated that CD36 overexpression enhances 
the Tyr416 phosphorylation of Src kinase in both cells (Figure 5A). Previous studies suggested that the activation of Src kinase could enhance calcium released from the endoplasmic reticulum pool to increase the free intracellular calcium concentrations by an inositol 1,4,5triphosphate(IP3)-dependent mechanism [24]. Here, CD36 overexpression elevates the levels of cytoplasm IP3 compared with vector control cells (Figure 5B). We further investigated the role of Src kinase in the CD36-induced HBV replication using a selective inhibitor of Src kinase SU6656. Our data showed that the Src kinase inhibitor SU6656 significantly diminished the CD36-induced increases of IP3, cytosolic calcium and HBV replication in both CD36 overexpression cells (Figure 5B, 5C and 5D). These results suggest that CD36 overexpression facilitated the phosphorylation of Src kinase to regulate HBV replication.

\section{Discussion}

$\mathrm{HBV}$ is an enveloped DNA virus that requires host lipid for the viral envelope and surface antigen particles $[25,26]$. The dysregulated lipid profile and altered lipid-related genes are likely due to metabolic hijacking by $\mathrm{HBV}$ in favor of its viral replication. A study demonstrated that fatty acid synthase (FAS) was upregulated in HBV-infected cells and the reduction of FAS by siRNA would suppress viral replication [27]. Recently, the relationship between fatty acid translocase CD36 and HBV has aroused our concern.

CD36 has been reported to facilitate Hepatitis C virus (HCV) entry in favor of viral replication, and blocking CD36 inhibited HCV replication [28]. Several studies have suggested that CD36 contributed to virion release in human immunodeficiency virus (HIV)-infected macrophages, and CD36 silenced or inhibited by soluble anti-CD36 antibodies showed 
decreasing quantities of HIV virions released [29]. Our previous study demonstrated that CD36 overexpression enhanced HBV replication in HepG2.215 cells [18]. Here, we found the same results in another HBV expression cell line, HepAD38 cells, which are derived from HepG2 and can replicate HBV under the control of the inducible tetracycline promoter. HepAD38 cells produce higher levels of viral DNA by approximately 11-fold compared with HepG2.2.15 cells [30]. Given these facts, an advantage of using HepAD38 cells is that HBV DNA replicative intermediates are easier to detect compared with HepG2.2.15 cells. This will be especially important in assessing antiviral effects in different cell lines to acquire relatively stable data, so the two cell lines were assessed in this study. We examined the effect of the depletion of CD36 on HBV replication and found that the suppression of CD36 possessed antiviral properties in both cell lines. These results suggest that the protein levels of CD36 are implicated in HBV replication in vitro.

HBV transgenic mice have been widely used in the functional studies of HBV replication and anti-HBV drugs $[31,32]$. However, the production of transgenic mice is very ambitious and expensive. Hydrodynamic injection is a rapid, efficient and convenient gene delivery method for in vivo transfection that has widely been used to generate acute HBV infection models via the hydrodynamic injection of HBV DNA plasmid [33,34]. In the present study, we successfully established an acute HBV infection mouse model via hydrodynamic injection and found that CD36 knockout mice had lower levels of HBV replication than wild type mice. The data corresponded with the cell experiments, strongly suggesting that CD36 plays an important role in regulating HBV replication.

With the development of transcriptome sequencing technology and bioinformatics, the 
RNA sequencing approach based on next-generation sequencing technology has been developed as a powerful tool for transcriptome analysis [35]. To understand the underlying molecular basis linking CD36 and HBV replication, we performed RNA sequencing of the mRNA from CD36 overexpression and the vector control HepG2.2.15 cells to analyze the differential transcriptomic profiles. Our results identified 141 differentially expressed genes in response to CD36 overexpression [18]. Unfortunately, the expression of cellular transcription factors related to HBV replication was not significantly different between the two groups. Then, we focused on some of the differentially expressed genes involved in ion channels and homeostasis. Our data suggested that CD36 overexpression affected ion homeostasis, especially calcium ion homeostasis, which plays an important role in HBV replication and assembly.

Calcium homeostasis is essential for maintaining normal cell function and small as the changes in cytosolic calcium levels might be affect the cellular processes [36]. Many viruses could regulate intracellular calcium signals by encoding virus proteins to stimulate the viral replication of HIV, adenovirus, and Rubella virus [37,38]. For HBV, various studies have suggested that $\mathrm{HBV}$ x protein $(\mathrm{HBx})$ elevated the basal cytosolic calcium level, which was associated with HBV replication in HepG2 cells and cultured primary rat hepatocytes $[39,40]$. The HBx-deficient sample significantly decreased HBV replication and could be rescued by simply increasing cytosolic calcium levels [19]. Although the viral-mediated regulation of $\mathrm{Ca}^{2+}$ levels has been associated with replicating $\mathrm{HBV}$, host mechanisms that affect $\mathrm{Ca}^{2+}$ signaling to regulate viral replication remain unknown. In this study, we found that CD36 expression could alter the cytosolic basal calcium levels, with a corresponding alternation of HBV replication in both HepG2.2.15 and HepAD38 cells. The calcium chelator BAPTA-AM could override the 
greater HBV replication increased by CD36 overexpression, and the calcium activator thapsigargin could improve the lower HBV replication reduced by CD36 knockdown. CD36 is just one of the calcium regulators, but CD36 knockdown could decrease the calcium concentrations by $29 \%$ and $18 \%$ in the HepG2.2.15 cells and HepAD38 cells respectively, with a corresponding decrease of $70 \%$ and $56 \%$ in HBV replication in the two cells. Similarly, Bouchard,et al $[19,41]$ found that one of the function of $\mathrm{HBx}$ was to regulate the cytosolic calcium, and the calcium levels was decreased by about $25 \%$ when $\mathrm{HBx}$ was deleted, consequently, HBV DNA replication was reduced by $95 \%$. These data suggest that HBV

leads to endoplasmic reticulum $\mathrm{Ca}^{2+}$ depletion and is followed by the triggering of calcium influx into cells to bring about a large increase in cytosolic calcium via SOC channels $[24,43]$. Several studies have suggested that HBx stimulates high levels of viral replication by stimulating polymerase activity via the activation of Src kinase and that blocking Src kinase could impair viral replication [44]. In this study, we found that high expression levels of CD36 improved the levels of Src kinase phosphorylation and the production of IP3. SU6656 as a commonly used inhibitor for Src replication is very sensitive to the changes in intracellular calcium levels.

In non-excitable cells, such as hepatocytes, increases in cytosolic calcium mainly originate from two sources: $\mathrm{Ca}^{2+}$ release from the endoplasmic reticulum $\mathrm{Ca}^{2+}$ stores by the activation of IP3 receptors and $\mathrm{Ca}^{2+}$ influx from outside the cell by opening store-operated $\mathrm{Ca}^{2+}$ (SOC) channels [42]. Multiple studies have revealed that linoleic acid or palmitate is implicated in the phosphorylation of Src kinase by binding to CD36. Consequently, this 
phosphorylation induces the production of IP3, which kinase, could inhibit phosphorylation of Src kinase and the following calcium signaling pathways [45]. The Src kinase inhibitor SU6656 reduced IP3 secretion and cytosolic calcium levels, with a corresponding decrease of $\mathrm{HBV}$ replication, suggesting that $\mathrm{CD} 36$ regulated $\mathrm{HBV}$ replication via the Src kinase phosphorylation pathway.

\section{Conclusions}

In general, we have characterized new aspects of fatty acid translocase CD36 biology in HBV replication by showing that CD36 overexpression and knockdown are positive and negative regulators of HBV replication via cytosolic calcium and the Src kinase pathway, respectively. Given the relationship between CD36 and HBV replication, we hypothesize that for those HBV-infected patients with a higher expression level of CD36, such as NAFLD and MS patients, targeting CD36 may serve as an adjunctive therapeutic means for conventional anti-viral drugs. For now, it is still unclear whether CD36 is involved in other steps of the HBV life cycle, including virus entry and secretion. Further experiments will be needed to reveal the contribution of CD36 to the HBV life cycle.

\section{Disclosure statement}

The authors have no financial or commercial conflict of interest.

\section{Acknowledgments}

We thank Dr. Maria Febbraio (Lerner Research Institute, U.S.) for providing the CD36 knockout mice. This study was supported by the National Natural Science Foundation of China 
(81570517, Key Program, No. 81390354, 31571210, 81400786, 81572683 and 81371827), Chongqing Research Program of Basic Research and Frontier Technology (No. cstc2015jcyjBX0044, cstc2015jcyjBX0011), and the Program for Innovation Team of Higher Education in Chongqing (grant no. CXTDX201601015).

\section{References}

[1] Ganem D, Prince AM. Hepatitis B virus infection--natural history and clinical consequences. N Engl J Med. 350 (2004) 1118-1129.

[2] Shlomai A, Shaul Y. The "metabolovirus" model of hepatitis B virus suggests nutritional therapy as an effective anti-viral weapon. Med hypotheses. 71 (2008) 53-57.

[3] Bar-Yishay I, Shaul Y, Shlomai A. Hepatocyte metabolic signalling pathways and regulation of hepatitis B virus expression. Liver Int. 31 (2011) 282-290.

[4] Evans AA, O'Connell AP, Pugh JC, et al. Geographic variation in viral load among hepatitis B carriers with differing risks of hepatocellular carcinoma. Cancer Epidemiology Biomarkers Pre. 7 (1998) 559-565.

[5] Shlomai A, Paran N, Shaul Y. PGC-1alpha controls hepatitis B virus through nutritional signals. Proc Natl Acad Sci USA. 103 (2006) 16003-16008.

[6] Kim HY, Cho HK, Choi YH, et al. Bile acids increase hepatitis B virus gene expression and inhibit interferon-alpha activity. FEBS J. 277 (2010) 2791-2802.

[7] Bremer CM, Bung C, Kott N, et al. Hepatitis B virus infection is dependent on cholesterol 
in the viral envelope. Cell Microbiol. 11 (2009) 249-260.

[8] Dorobantu C, Macovei A, Lazar C, et al. Cholesterol depletion of hepatoma cells impairs hepatitis B virus envelopment by altering the topology of the large envelope protein. J Virol. 85 (2011) 13373-13383.

[9] Wong GL, Wong VW, Choi PC, et al. Metabolic syndrome increases the risk of liver cirrhosis in chronic hepatitis B. Gut. 58 (2009) 111-117.

[10] Jarcuska P, Janicko M, Kruzliak P, et al. Hepatitis B virus infection in patients with metabolic syndrome: a complicated relationship. Results of a population based study. Eur J Intern Med. 25 (2014) 286-291.

[11] Silverstein R.L, Febbraio M. CD36, a scavenger receptor involved in immunity, metabolism, angiogenesis, and behavior. Sci Signal. 2 (2009) re3.

[12] Miquilena-Colina ME, Lima-Cabello E, Sanchez-Campos S, et al. Hepatic fatty acid translocase CD36 upregulation is associated with insulin resistance, hyperinsulinaemia and increased steatosis in non-alcoholic steatohepatitis and chronic hepatitis C. Gut 60 (2011) 13941402.

[13] Koonen DP, Jacobs RL, Febbraio M, et al. Increased hepatic CD36 expression contributes to dyslipidemia associated with diet-induced obesity. Diabetes 56 (2007) 2863-2871.

[14] Handberg A, Norberg M, Stenlund H, et al. Soluble CD36 (sCD36) clusters with markers of insulin resistance, and high sCD36 is associated with increased type 2 diabetes risk. J Clin Endocrinol Metab. 95 (2010) 1939-1946. 
[15] Heebøll S, Poulsen MK, Ornstrup MJ, et al. Circulating sCD36 levels in patients with nonalcoholic fatty liver disease and controls. Int J Obes (Lond). 41 (2017) 262-267.

[16] Schoeman JC, Hou J, Harms AC, et al. Metabolic characterization of the natural progression of chronic hepatitis B. Genome Med. 8 (2016) 64.

[17] Li H, Zhu W, Zhang L, et al.The metabolic responses to hepatitis B virus infection shed new light on pathogenesis and targets for treatment. Sci Rep. 5 (2015) 8421.

[18] Huang J, Zhao L, Yang P, et al. Genome-Wide Transcriptome Analysis of CD36 Overexpression in HepG2.2.15 Cells to Explore Its Regulatory Role in Metabolism and the Hepatitis B Virus Life Cycle. PLoS One 11 (2016) e0164787.

[19] Bouchard MJ, Wang LH, Schneider RJ. Calcium signaling by HBx protein in hepatitis B virus DNA replication. Science 294 (2001) 2376-2378.

[20] Choi Y, Gyoo Park S, Yoo JH, Jung G. Calcium ions affect the hepatitis B virus core assembly. Virology 332 (2005) 454-463.

[21] Bull HA, Brickell PM, Dowd PM. Src-related protein tyrosine kinases are physically associated with the surface antigen CD36 in human dermal microvascular endothelial cells. FEBS Lett. 351 (1994) 41-44.

[22] Kuda O, Jenkins CM, Skinner JR, et al. CD36 protein is involved in store-operated calcium flux, phospholipase A2 activation, and production of prostaglandin E2. J Biol Chem. 286 (2011) 17785-17795. 
[23] Roskoski R Jr. Src kinase regulation by phosphorylation and dephosphorylation. Biochem Biophys Res Commun. 331 (2005) 1-14.

[24] El-Yassimi A, Hichami A, Besnard P, et al. Linoleic acid induces calcium signaling, Src kinase phosphorylation, and neurotransmitter release in mouse CD36-positive gustatory cells. J Biol Chem. 283 (2008) 12949-12959.

[25] Gavilanes F, Gonzalez-Ros JM, Peterson DL, et al.Structure of hepatitis B surface antigen. Characterization of the lipid components and their association with the viral proteins. J Biol Chem. 257 (1982) 7770-7777.

[26] Satoh O, Umeda M, Imai H, et al. Lipid composition of hepatitis B virus surface antigen particles and the particle-producing human hepatoma cell lines. J Lipid Res. 31 (1990) 12931300.

[27] Zhang H, Li H, Yang Y, et al. Differential regulation of host genes including hepatic fatty acid synthase in HBV-transgenic mice. J Proteome Res. 12 (2013) 2967-2979.

[28] Cheng JJ, Li JR, Huang MH, et al. CD36 is a co-receptor for hepatitis C virus E1 protein attachment. Sci Rep. 6 (2016) 21808.

[29] Berre S, Gaudin R, Cunha de Alencar B, et al. CD36-specific antibodies block release of HIV-1 from infected primary macrophages and its transmission to T cells. J Exp Med. 210 (2013) 2523-2538.

[30] Ladner SK, Otto MJ, Barker CS, et al. Inducible expression of human hepatitis B virus (HBV) in stably transfected hepatoblastoma cells: a novel system for screening potential 
inhibitors of HBV replication. Antimicrob Agents Chemother. 41 (1997) 1715-1720.

[31] Chisari FV. Hepatitis B virus transgenic mice: insights into the virus and the disease. Hepatology 22 (1995) 1316-1325.

[32] Di Scala M, Otano I, Gil-Fariña I, et al.Complementary Effects of Interleukin-15 and Alpha Interferon Induce Immunity in Hepatitis B Virus Transgenic Mice. J Virol. 90 (2016) 85638574.

[33] Yang PL, Althage A, Chung J, Chisari FV. Hydrodynamic injection of viral DNA: A mouse model of acute hepatitis B virus infection. Proc Natl Acad Sci USA. 99 (2002) 13825-13830.

[34] Liu FJ, Liu L, He F, et al. Establishment and primary application of a mouse model with hepatitis B virus replication. World J Gastroenterol. 13 (2007) 5324-5330.

[35] Wang Z, Gerstein M, Snyder M. RNA-Seq: a revolutionary tool for transcriptomics. Nat Rev Genet. 10 (2009) 57-63.

[36] Carafoli E, Santella L, Branca D, Brini M. Generation, control, and processing of cellular calcium signals. Crit Rev Biochem Mol Biol. 36 (2001)107-260.

[37] Chami M, Oules B, Paterlini-Brechot P. Cytobiological consequences of calcium-signaling alterations induced by human viral proteins. Biochim Biophys Acta 1763 (2006) 1344-1362.

[38] Zhou Y, Frey TK, Yang JJ. Viral calciomics: interplays between $\mathrm{Ca}^{2+}$ and virus. Cell Calcium 46 (2009) 1-17.

[39] Casciano JC, Duchemin NJ, Lamontagne RJ, et al. Hepatitis B virus modulates store- 
operated calcium entry to enhance viral replication in primary hepatocytes. PLoS One 12 (2017) $\mathrm{e} 0168328$.

[40] Yang B, Bouchard MJ. The hepatitis B virus X protein elevates cytosolic calcium signals by modulating mitochondrial calcium uptake. J Virol. 86 (2012) 313-327.

[41] McClain SL, Clippinger AJ, Lizzano R, Bouchard MJ. Hepatitis B virus replication is associated with an $\mathrm{HBx}$-dependent mitochondrion-regulated increase in cytosolic calcium levels. J Virol. 81 (2007) 12061-12065.

[42] Tojyo Y, Morita T, Nezu A, Tanimura A. Key components of store-operated $\mathrm{Ca}^{2+}$ entry in non-excitable cells. J Pharmacol Sci. 125 (2014) 340-346.

[43] Xu S, Nam SM, Kim JH, et al. Palmitate induces ER calcium depletion and apoptosis in mouse podocytes subsequent to mitochondrial oxidative stress. Cell Death Dis. 6 (2015) e1976.

[44] Klein NP, Bouchard MJ, Wang LH, et al. Src kinases involved in hepatitis B virus replication. EMBO J. 18 (1999) 5019-5027.

[45] Suresh K, Servinsky L, Reyes J, et al. Hydrogen peroxide-induced calcium influx in lung microvascular endothelial cells involves TRPV4. Am J Physiol Lung Cell Mol Physiol. 309 (2015) $1467-1477$.

Fig. 1. Overexpression of CD36 promoted HBV replication. (A) Western blotting and 
quantitative analysis of CD36 protein in HepG2.2.15 and HepAD38 cells transfected with human CD36 overexpression lentivirus $(\mathrm{CD} 36 \mathrm{OE})$ or vector $(\mathrm{n}=3)(* * * \mathrm{p}<0.001)$. Actin was used as a loading control. (B) Core particles associated with HBV DNA were extracted from $\mathrm{CD} 36 \mathrm{OE}$ and vector cells after transfection for 4 days and were identified by southern blot. $\mathrm{M}$, marker; rcDNA: intracellular HBV relax circle; ssDNA: single-stranded DNA. (C) HBV DNA levels were also determined by real-time PCR $(n=4)$. The secretion levels of HBeAg and HBsAg in culture medium of CD36OE and vector cells in HepG2.2.15 and HepAD38 cells were collected and measured using ELISA. O.D. values were expressed as a percentage relative to the vector $(\mathrm{n}=3)(* \mathrm{P}<0.05, * * \mathrm{P}<0.01)$.

Fig. 2. Gene silencing of CD36 inhibited HBV replication. (A) Western blotting and quantitative analysis of the CD36 protein in HepG2.2.15 and HepAD38 cells transfected with lentiviruses expressing shRNA-targeting CD36 (shCD36) or shRNA-scramble control (shCont) $(\mathrm{n}=3)(* * * \mathrm{p}<0.001)$. Actin was used as a loading control. (B) Core particles associated with HBV DNA were extracted from shCD36 and shCont cells after transfection for 4 days and were identified by southern blot. M, marker; rcDNA: intracellular HBV relax circle; ssDNA: singlestranded DNA. (C) HBV DNA levels were also determined by real-time PCR $(n=4)$. The secretion levels of HBeAg and HBsAg in culture medium of shCD36 and shCont in HepG2.2.15 and HepAD38 cells were collected and measured using ELISA. O.D. values were expressed as a percentage relative to the shCont $(\mathrm{n}=5) .(* \mathrm{P}<0.05, * * \mathrm{P}<0.01$ and $* * * \mathrm{p}<0.001)$. (D) The levels of $\mathrm{HBs} \mathrm{Ag}$ and $\mathrm{HBe} \mathrm{Ag}$ in serum of $\mathrm{HBV}$-infected WT and CD36KO mice by chemiluminescence immunoassay $(n=3)$. HBV DNA levels in the serum and liver were detected by real-time $\mathrm{PCR}(\mathrm{n}=4)(* \mathrm{P}<0.05, * * \mathrm{P}<0.01)$. HBV core protein expression in the liver of HBV- 
infected WT and CD36KO mice was visualized by immunohistochemistry using anti-HBcAg; arrows indicate $\mathrm{HBcAg}$.

Fig. 3. Effects of CD36 expression on cytosolic calcium levels. (A) Differentially expressed genes from RNA sequencing analysis were involved in ion homeostasis in HepG2.2.15CD36OE and HepG2.2.15-vector cells. (B) Overexpression of CD36 in HepG2.2.15 and HepAD38 cells increases cytosolic $\mathrm{Ca}^{2+}$. HepG2.2.15-CD36OE, HepAD38-CD36OE and vector cells were loaded with the fluorescent $\mathrm{Ca}^{2+}$ indicator Fluo-4/AM after culture for $48 \mathrm{~h}$ and measured using flow cytometry. $\mathrm{Ca}^{2+}$ concentrations are expressed as relative fluorescence $(\mathrm{n}=3)(* \mathrm{p}<0.05, * * \mathrm{P}<0.01)$. (C) Knockdown of CD36 in HepG2.2.15 and HepAD38 cells decreases cytosolic $\mathrm{Ca}^{2+}$. HepG2.2.15 and HepAD38 cells were transfected with CD36shRNA-lentivirus (shCD36) and scramble control-lentivirus (shCont), treated with $4 \mu \mathrm{M}$ Fluo4/AM after $48 \mathrm{~h}$ posttransfection, and measured using flow cytometry. $\mathrm{Ca}^{2+}$ concentrations are expressed as relative fluorescence $(n=3)(* * p<0.01)$.

Fig. 4. CD36 regulation of $\mathrm{HBV}$ replication is associated with cytosolic calcium signals. (A) Calcium chelator (BAPTA-AM) reduces HBV replication in CD36OE of HepG2.2.15 and HepAD38 cells. BAPTA-AM or vehicle controls (DMSO) were added to the CD36OE of HepG2.2.15 and HepAD38 cells for $96 \mathrm{~h}$, and HBV DNA were extracted and detected by Realtime PCR ( $\mathrm{n}=4)$. Cytosolic $\mathrm{Ca}^{2+}$ was measured after treatment for $48 \mathrm{~h}$ using flow cytometry (n=3). (B) Calcium activator (thapsigargin) rescued HBV replication in CD36 knockdown HepG2.2.15 and HepAD38 cells. Thapsigargin (TG) or vehicle controls (DMSO) were added to the CD36 knockdown HepG2.2.15 and HepAD38 cells for 96 h, and HBV DNA were 
extracted and detected by real-time PCR $(n=4)$. Cytosolic $\mathrm{Ca}^{2+}$ was measured after being treated for $48 \mathrm{~h}$ using flow cytometry $(\mathrm{n}=3) .\left(* * \mathrm{P}<0.01\right.$ and $\left.{ }^{* * *} \mathrm{p}<0.001\right)$.

Fig. 5. CD36 overexpression activated Src kinase, and Src inhibitors override the higher HBV replication induced by CD36 overexpression. (A) CD36 overexpression activated Src kinase in HepG2.2.15 and HepAD38 cells. The cell protein was collected from CD36overexpressing HepG2.2.15 and HepAD38 cells after culture for 72 h. Total Src and the phosphorylation levels were detected using western blotting $(\mathrm{n}=3)\left(* * \mathrm{P}<0.01\right.$ and $\left.^{* * *} \mathrm{p}<0.001\right)$. (B) IP3 levels measured by ELISA in CD36OE and vector control of HepG2.2.15 and HepAD38 cells $(\mathrm{n}=3)(* \mathrm{P}<0.05)$. (C) Src kinase inhibitor (SU6656) decreases cytosolic $\mathrm{Ca}^{2+}$ in CD36OE of HepG2.2.15 and HepAD38 cells. SU6656 or vehicle controls (DMSO) were added to the CD36OE of HepG2.2.15 and HepAD38 cells for 24h, Cytosolic $\mathrm{Ca}^{2+}$ was measured after treatment using flow cytometry $(n=3)(* * * p<0.001)$. (D) Src kinase inhibitor (SU6656) overrides the higher HBV replication induced by CD36 overexpression. SU6656 or vehicle controls (DMSO) were added to the CD36 overexpression of HepG2.2.15 and HepAD38 cells for 96 h. HBV DNA were extracted and detected by real-time PCR $(n=4)$. $\left(* * \mathrm{P}<0.01\right.$ and $\left.^{* * *} \mathrm{p}<0.001\right)$. 